\title{
Marketing digital al servicio de la gestión universitaria. Caso de estudio: la carrera ingeniería de sistemas de la UNESUM, Ecuador
}

\section{Digital marketing at the service of university management. Case study: the Systems Engineering career at UNESUM, Ecuador}

\section{Marketing digital al servicio de la gestión universitaria}

\author{
Karina Virginia Mero Suárez ${ }^{(1)}$ \\ Edwin Joao Merchán Carreño ${ }^{(2)}$ \\ Carlos Renán Mero Suárez ${ }^{(3)}$
}

(1) Universidad Estatal del Sur de Manabí, Manabí, Ecuador. email: karinaunesum@yahoo.com

(2) Universidad Estatal del Sur de Manabí, Manabí, Ecuador. email: joaounesum@yahoo.es

(3) Universidad Estatal del Sur de Manabí, Manabí, Ecuador. email: carlos_mero_1980@hotmail.com

Contacto: carlos_mero_1980@hotmail.com

Recibido: 30-04-2020

Aprobado: 08-06-2020

\begin{abstract}
Resumen
La educación superior se ha visto en la necesidad de adoptar nuevas prácticas de publicidad como respuesta a los múltiples cambios políticos, tecnológicos y sociales, así como al aumento de la competencia y la internacionalización. El presente trabajo tiene como propósito valorar las potencialidades tecnológicas, la factibilidad y disposición de los docentes y gestores de la carrera Ingeniería en Sistemas Computacionales para integrar el marketing digital a su estrategia de visualización de resultados y ofertas en la Universidad Estatal de Sur de Manabí en Ecuador, para ello se encuestaron 31 docentes, de ellos 1 Doctor en Ciencias, 30 Másters y 2 con cargos de dirección y gestión universitaria. Los resultados obtenidos permiten determinar la factibilidad de utilizar el marketing digital en la gestión de los procesos universitarios, como vía para mostrar las opciones educativas a los diferentes segmentos de la población, con el apoyo de la web social. El proceso de diagnóstico facilitó el análisis del proceso de marketing y las necesidades de capacitación de los docentes y administrativos.
\end{abstract}

Palabras clave: marketing digital, gestión universitaria, redes sociales, carrera Ingeniería en Sistemas Computacionales.

\footnotetext{
Abstract

Higher education has seen the need to adopt new advertising practices as a response to multiple political, technological and social changes, as well as to increased competition and internationalization. The purpose of this work is to assess the technological potentials, the feasibility
}

and willingness of teachers and managers of the Computer Systems Engineering degree to integrate digital marketing into their strategy for displaying results and offers at the State University of Sur de Manabí in Ecuador, 31 teachers were surveyed, 1 Doctor of Science, 30 Masters and 2 with management and university management positions. The results obtained allow us to determine the feasibility of using digital marketing in the management of university processes, as a way to show educational options to different segments of the population, with the support of the social web. The diagnostic process facilitated the analysis of the marketing process and the training needs of teachers and administrators.

Keywords: digital marketing, university management, social networks, career in Computer Systems Engineering.

\section{Introducción}

La publicidad ha experimentado en la última década cambios importantes que no solo han afectado al modo de conceptualizar, planificar y desarrollar los mensajes, sino que incluso han llegado a cuestionar la propia función y objetivos de la misma. Esto ha sido provocado por el desarrollo tecnológico y social liderado por Internet que, en el marco de la sociedad de la información, ha modificado las formas de comunicación, de relacionarse, de trabajar y colaborar.

En este escenario, el marketing digital combina los principios del marketing convencional con las oportunidades y posibilidades que brinda Internet y la interactividad de los servicios que ofrece. Los 
cambios en el mercado implican la necesidad de dominar nuevos medios para aprovechar todo su potencial y llegar a la consecución de los objetivos en las empresas, organizaciones e instituciones, independientemente de su objeto social. Entre las áreas del marketing digital se incluye el posicionamiento en buscadores, medición y análisis del tráfico en un web site, campañas de email, publicidad en buscadores $\mathrm{y}$ on line, desarrollo y diseño de sitios web, comercio electrónico, desarrollo de aplicaciones (intranets, extranets), marketing viral y buzz marketing, blogs, redes sociales, entre otros (Sosa y Useche, 2017).

La UNESCO (1998), afirma que "las instituciones universitarias están obligadas a implementar modernos enfoques de dirección en su práctica diaria y a utilizar los recursos de que disponen con gran eficacia y eficiencia. Estas circunstancias, así como las que se derivan de la necesidad de una mayor coordinación y racionalidad para dar respuesta a los nuevos requerimientos que imponen las exigencias multidisciplinarias de los procesos y de una mayor competencia por el uso de recursos comunes, le imprimen un sello característico a la gestión universitaria de estos tiempos". En esta investigación se coincide con Toledo y Luque (2017), cuando plantea que las universidades deben aplicar medidas de marketing en su gestión, como respuesta a los múltiples cambios políticos, tecnológicos y sociales que afecta al mundo de la educación superior, trayendo consigo el aumento de la competencia y la internacionalización.

Basados en este contexto, el presente trabajo propone valorar las potencialidades tecnológicas, la factibilidad y disposición de los docentes y gestores de la carrera Ingeniería en Sistemas Computacionales para integrar el marketing digital a su estrategia de visualización de resultados y ofertas en la Universidad Estatal de Sur de Manabí en Ecuador.

\section{Materiales y métodos}

La investigación se desarrolló en la carrera Ingeniería en Sistemas Computacionales de la Universidad Estatal del Sur de Manabí, Ecuador. Se realizó el muestro a 31 docentes, de ellos 2 ocupan cargos administrativos, 8 son mujeres y 23 hombres. El claustro está conformado por 31 Máster y 1 doctor en Ciencias. La carrera tiene a su disposición tecnología que permite el uso de herramientas de marketing digital.

La investigación utilizó un diseño no experimental, del tipo exploratorio y descriptivo, como métodos el deductivo, el análisis-síntesis para determinar el nivel de conocimiento y competencias que los docentes tenían sobre el marketing digital y las herramientas más factibles de utilizar. Se valoró la disposición de los docentes y gestores de la carrera para integrar el marketing digital a su estrategia de visualización de resultados y ofertas. La investigación se realizó de enero a marzo 2019.

\section{Desarrollo}

El impacto de la globalización en las universidades se caracteriza por el incremento de los índices de matriculación, menor financiación pública y la aparición del paradigma del aprendizaje a lo largo de la vida (Dale, 2007). Este fenómeno y la búsqueda continua para mejorar la gestión y el servicio universitario, ha provocado que en los últimos años las universidades hayan adquirido nuevas tácticas, desde una perspectiva del marketing, que le permita actuar de forma más eficaz y planificada, así como adaptarse al nuevo entorno competitivo.

Para Toledo y Luque (2017), entro los principales motivos por los cuales las universidades comenzaron a aplicar, de forma más explícita, conceptos propios de marketing se pueden destacar los siguientes:

- Nueva gestión pública: las universidades aplican cada vez más políticas supranacionales (por ejemplo, en Europa) y existe una creciente competencia entre ellas.

- Masificación: la población universitaria mundial se duplicó entre 1975 y el año 2000.

- $\quad$ Profesionalización de la enseñanza superior: preparar adecuadamente a los estudiantes para el mercado de trabajo.

- $\quad$ Reducción de la financiación pública.

- Toma de conciencia de problemas de gestión de la calidad

- El avance en la gestión de los servicios.

Por otro lado, las universidades se han trasformado considerablemente, poseen un mayor grado de participación en la política, la economía, el deporte y la cultura, lo que ha tenido como resultado que se reconozcan, no solo en su medio académico, sino en las sociedades a nivel mundial. Los espacios universitarios se hallan cada vez más impactados por las tecnologías que ofrecen nuevas oportunidades dentro de los procesos de enseñanza-aprendizaje, y que a su vez forman diferentes plataformas para publicitar y visibilizar las instituciones.

Dada la naturaleza cambiante del sector de la educación superior, es aconsejable que los gerentes y líderes de las universidades adopten un enfoque centrado en el usuario. Cada vez más, estas 
instituciones se reconocen como una industria de servicios y, por lo tanto, ponen mayor énfasis en la satisfacción del cliente y en una adecuada gestión de la universidad (Deshields et al., 2005). Tradicionalmente, las instituciones de educación superior han carecido de una consolidada cultura de gestión, sin embargo, la mayoría de ellos aplican estrategias con este fin para lograr niveles de comercialización diariamente, a menudo inconscientemente, y sin un conocimiento preciso del proceso que realizan (Kirp, 2013; Siu y Wilson, 2017).

Cuando las universidades crean perfiles en redes sociales, y éstos tienen una interacción exitosa con sus seguidores dentro de la comunidad universitaria, los buscadores mejoran la posición de las páginas web a las que se alude en las publicaciones. De esta manera, cuando un usuario busca información relacionada con estos centros los resultados aparecen en las primeras posiciones (Cordero, 2016; Ayobí, 2017).

Con el objetivo de crear una ventaja competitiva sostenible en el entorno actual de las instituciones de educación superior, Arnoldo y Nicolás (2011), consideran que una adecuada gestión de la universidad debe comprender las siguientes estrategias:

1. estrategia financiera para una asignación y aprovechamiento de los recursos

2. estrategia de recursos humanos, que incluye tanto a los docentes como al personal administrativo

3. estrategia tecnológica, en este concepto se incluye, por ejemplo, el equipamiento audiovisual de las salas de clases, bibliotecas y equipamiento de laboratorios

4. estrategia de adquisiciones, que se aplica a todo lo relacionado con el área de adquisiciones para la operación interna

5. estrategia de servicio, que define los objetivos estratégicos del producto o servicio que la unidad entrega en términos de costo, calidad, confiabilidad, flexibilidad e innovación

6. estrategia de marketing, que promueve las políticas de la institución respecto a las acciones para captar alumnos de pregrado y postgrado, tanto nacionales como internacionales, y anunciarse de manera propicia al medio externo

A la hora de implementar una estrategia de marketing en el ámbito universitario el principal escollo que aparece es la reducida concepción de los responsables de la gestión de mercado. Existen instituciones (públicas mayormente) que se resisten a la filosofía del marketing porque la asocian al mundo de los negocios dada su naturaleza comercial, creyendo que solo sirve para captar estudiantes y su consecuente generación de ingresos, sin analizar que el reto consiste en la contradicción de la filosofía del marketing en las universidades y su verdadera aplicación. Es por ello que el principal desafío del marketing en el sector universitario es la resistencia a su implantación y puesta en práctica (Hayes, 2016).

Son diversas las variables que influyen en la toma de decisión que el estudiante y/o su familia efectúa en el momento de optar por el ingreso a una universidad que aparece como oferta, para muchos autores, la más influyente es el prestigio de la institución. Weber (1962), identifica el prestigio como uno de los elementos clave para poseer poder social. El prestigio, al igual que el poder, está desigualmente distribuido. Esta acumulación desigual de prestigio habla de posiciones en una estructura y, es así, como puede referirse a la estructura del mercado universitario.

Zapata (2007), recomendó como posibles alternativas de comunicación:

- Publicidad: a través de medios como prensa y revistas especializadas.

- Relaciones públicas: Presentaciones de investigaciones y publicaciones de la especialización en foros y encuentros internacionales, nacionales y regionales.

- Marketing on line: divulgación de programas en Internet.

- Ventas personales: establecer un grupo de promotores directos de los programas, que colaboren en la consecución de candidatos, se sugiere que hayan sido egresados.

- Folletos y plegables promocionales: con testimonios de egresados exitosos de los programas ofrecidos.

- Presentación de programas dirigidos a interesados en el que participen docentes, egresados y directivos junto con ejecutivos de empresas que hayan vinculado en sus organizaciones egresados de la universidad

En este sentido, una óptica orientada al marketing se presenta como un recurso fundamental para crear una ventaja sostenible en el tiempo, mantener y mejorar la producción. Según Franco y Arrubla (2011) y Hult y Ketchen (2018), esta estrategia se ha intentado aplicar desde el mundo académico, sobretodo, desde principios de los años ochenta del siglo pasado, especialmente en universidades norteamericanas (Bardo et al., 2014). 
La inversión publicitaria en medios es una más de las tantas estrategias de marketing que ejecutan las universidades. La construcción de la imagen y la marca de la institución se llevan a cabo a través de múltiples medios y técnicas. El marketing digital es una forma de mercadeo que utiliza medios tecnológicos, para proyectar la imagen de una marca, mediante la publicidad de productos $\mathrm{y}$ servicios de una empresa, negocio y/o organización en diferentes canales de Internet, bien sean redes sociales, páginas web, radio online, plataformas de vídeo y televisión, entre otras. La inversión en publicidad está constituida por acciones tales como el diseño y distribución de papelería, las charlas vocacionales, las giras de promoción nacionales, el seguimiento de egresados, la extensión cultural (Wörner, 2016).

\section{Resultados}

En la Universidad Nacional Estatal del Sur de Manabí (UNESUM) en la República de Ecuador, se desarrolla la carrera Ingeniería en Sistemas Computacionales, que cuenta con un claustro de 31 profesores, de ellos 1 Doctor en Ciencias, 30 Másters y 2 ocupan cargos de dirección y gestión universitaria. Cuenta con una matrícula de 649 estudiantes. En esta investigación se valora la efectividad de usar las herramientas de marketing digital, para alcanzar mayores niveles de gestión y visualización de los servicios que la carrera ofrece a la sociedad.

Se realiza una encuesta en la que se comprueba que solo el $2.79 \%$ de los docentes usan las herramientas de la web 2.0 para la publicidad y visualización de las ofertas educativas de la carrera. La causa fundamental está en la concepción generalizada que el marketing, y en especial el digital, es más útil en el contexto empresarial, a pesar de que el $80.64 \%$ tiene conocimiento de las aplicaciones más usadas en el mundo para este fin.

De ahí que se les consulte su criterio sobre la utilización de algunas herramientas para desarrollar la gestión de marketing digital en la carrera. Los resultados que se muestran en el gráfico 1 permiten comprobar que 26 (para un $83.87 \%$ ) consideran las redes sociales como la opción idónea.

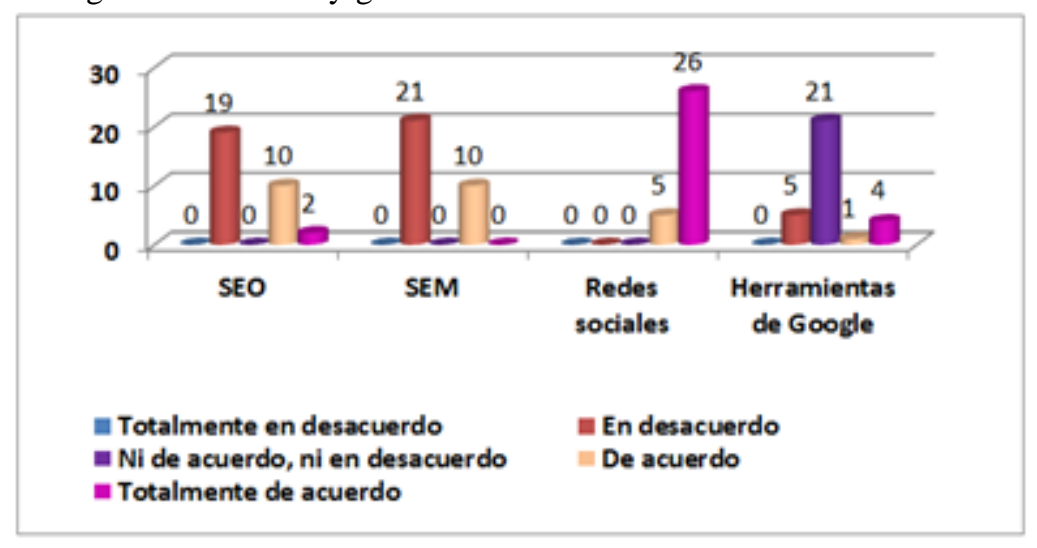

Gráfico 1. Herramientas para el marketing digital.

Así mismo, se consultó a los docentes sobre las redes sociales que podían ser usadas para el proceso de publicidad de la carrera Ingeniería en Sistemas Computacionales. Se determina que la causa fundamental de la selección está en el dominio que los docentes tienen de las mismas, no de la factibilidad que tengan para su uso en la función del marketing digital (Gráfico 2). El $54.83 \%$ de los docentes (17) apuestan por el uso de Facebook, seguida de Linkedin $(8,25.80 \%)$ y ResearchGate $(3,9.67 \%)$.

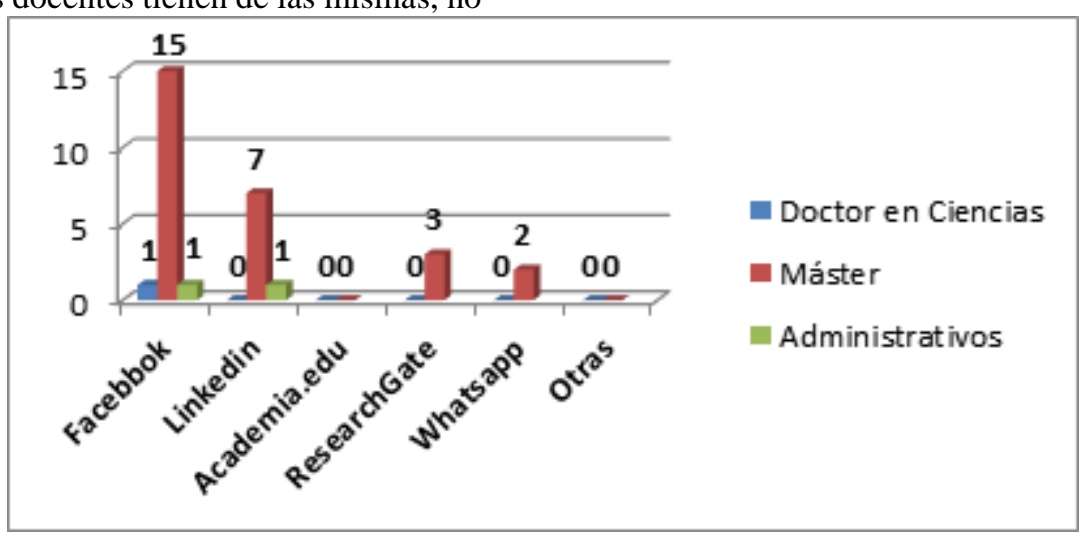


Gráfico 2. Redes sociales seleccionadas para el marketing digital.

Una vez comprobado el interés por el uso de estas herramientas para alcanzar mejores resultados en la promoción de los productos y servicios de la carrera, se pasa a comprobar las necesidades de capacitación para llevar a cabo la propuesta. En el gráfico 3, se observa que las opciones más requeridas, en las que el $100 \%$ de los docentes coinciden, son el diseño de páginas institucionales en redes sociales, la promoción de productos y servicios y el diseño de infografías; seguidas de la segmentación de público destinatario, en la que concuerdan los doctores y administrativos en un $100 \%$ de y el $87.09 \%$ de los Máster. La creación de grupos temáticos se considera importante por el Doctor en Ciencias y el $80.64 \%$ de los Máster en Ciencias, los administrativos no consideran necesaria esta preparación. Los temas de la imagen y el diseño del texto para la publicidad, tuvieron una menor solicitud. Estos resultados exigirán un diseño adecuado de la formación a los docentes, que permita una capacitación personalizada, según las funciones de cada uno.

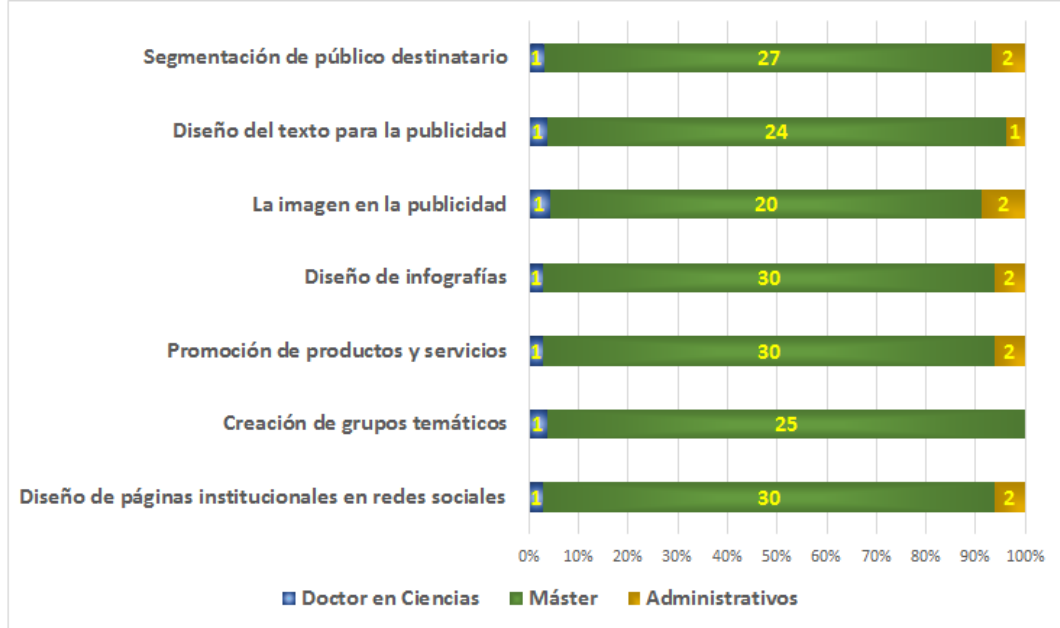

Gráfico 3. Necesidades de capacitación en redes sociales para el marketing digital en la carrera Ingeniería en Sistemas Computacionales de la UNESUM.

El resultado de estas encuestas permite comprobar que los docentes y personal administrativo, considera que las redes sociales cumplen una función personal, social y de información comercial y académica, lo que elevaría la calidad del proceso que en la universidad y la carrera se desarrolla.

\section{Discusión}

Coincidiendo con estos resultados, Lahuerta y Cordero (2016), los encuestados consideran que los usuarios de redes sociales construyen un perfil personal que les permite comentar, expresarse, o compartir contenidos (fotos, vídeos, estados o comentarios) que se convierten en públicos y por tanto van más allá de su privacidad. Dichos perfiles constituyen la base del funcionamiento de las redes sociales. Estas plataformas son concebidas como un entorno de intercambio social, son espacios virtuales donde cada miembro puede compartir e intercambiar con otros sus opiniones, intereses y experiencias de todo tipo.

Otra de sus características es la opción de encontrar a amigos o usuarios con perfiles similares que permiten ampliar la red de contactos del individuo y por tanto su sociabilidad. Este hecho implica que el contenido difundido por un usuario aumente su alcance más allá de sus contactos. Por último, las redes sociales permiten la transferencia de información con y entre empresas, ya que dichos espacios virtuales permiten el contacto directo y dinámico entre las organizaciones y otros usuarios que pueden ser o llegar a ser clientes.

En la investigación desarrollada por estos autores se comprueba que, la presencia de las universidades en estas redes ha hecho que estas instituciones hayan encontrado diversas utilidades de las plataformas en su propio beneficio. Lavrusik (2009), considera, además, que con ellas se puede:

- Recolectar y diseminar información. La red es un excelente medio para difundir noticias, y para mostrar al público los recursos y los expertos con los que cuenta la universidad.

- Dar a conocer el trabajo de los estudiantes y de las facultades. Las redes sociales pueden servir de vehículo para comunicar las últimas novedades, logros o premios de una facultad, y los propios estudiantes pueden también compartir sus fotografías, vídeos o experiencias relacionadas con las asignaturas de su carrera. 
- Proporcionar una plataforma para la difusión de eventos. Las universidades pueden hacer uso de los medios sociales (por ejemplo, proyectar vídeos en directo a través de un canal de YouTube para dar difusión internacional a sus eventos más destacados (la inauguración de un curso académico, una investidura honoris causa, entre otras).

- Conectar personas. Los nuevos estudiantes, pueden hacer amigos y recibir consejos de los alumnos más veteranos. A su vez, los alumnos pueden establecer conexiones entre sí que mejoren sus recursos a la hora de realizar trabajos o investigaciones. Estas relaciones se pueden extender igualmente al profesorado $\mathrm{y}$ al personal de administración y servicios.

- Producir, no solo promocionar. Las plataformas de redes sociales horizontales permiten a las universidades producir y distribuir contenidos adicionales (entrevistas, vídeos, posts, difusión de noticias y documentación adicional) para acercarse a toda la comunidad educativa de una manera amena y dinámica, evitando las rigideces del sistema tradicional de difusión de noticias y comunicados.

- Crear un diálogo para comunicarse con los estudiantes. Las tecnologías 2.0 permiten la característica única de una comunicación bi-direccional entre la universidad y su público, y además facilita las conversaciones en tiempo real. Los estudiantes pueden interactuar preguntando, respondiendo o participando en la co-creación de contenidos relevantes para la comunidad en tiempo real (por ejemplo, la creación de una wiki o la discusión en un foro de debate en línea).

Así mismo, Kotler y Armstrong (2007), señalan que el beneficio del marketing digital en la comunicación interactiva es la inmediatez, mayor retroalimentación, comunicación, instantaneidad e interacción. En esta investigación los docentes y personal de gestión universitaria coincidieron en que, gracias a la implementación de los medios del marketing digital, el estudiante siempre estará informado sobre la situación actual de la institución y esto ayudará a resolver problemas, en muchos casos, sin necesidad que el estudiante se traslade a la universidad.

Muñiz (2010), manifiesta las ventajas para el usuario del uso de un medio digital para conseguir información necesaria. En los resultados en materia promocional a través del marketing digital, los entrevistados manifestaron que entre los beneficios se encuentran: menor inversión en medios tradicionales, mayor visibilidad, mayor alcance y aumento de clientes. Así mismo, se constató el beneficio que pueden observar es que cada vez son más los estudiantes que solicitan información y la respuesta es más efectiva gracias a la implementación marketing digital.

Sosa y Useche (2017), presentan una investigación sobre los medios utilizados en el marketing digital por las universidades privadas en el municipio Maracaibo del estado Zulia en Venezuela. La totalidad de los entrevistados manifestaron que el propósito es tener la información más ampliada para toda la comunidad estudiantil, que favorece diferentes tipos de informaciones, así como varios servicios al mismo tiempo (horario, pensum, inscripciones) de esta manera los diferentes tipos de públicos como: personal administrativo, estudiantes, personal obrero y público potencial se benefician.

Estas investigaciones coinciden con los resultados y visión de los encuestados (docentes, investigadores y personal administrativo) de la carrera Ingeniería en Sistemas Computacionales de la Universidad Estatal de Sur de Manabí en Ecuador, sobre el uso de las redes sociales. El uso de las herramientas de marketing digital es de vital importancia, ya que brindan excelentes oportunidades para realizar publicidad, incentivan la interacción con los clientes y promueve la adquisición de un producto o servicio. Al utilizar las bondades que ofrecen dichas herramientas se alcanza una importante forma de hacer publicidad a menor costo y de llegar a más clientes potenciales.

\section{Conclusiones}

Los resultados obtenidos permiten determinar la factibilidad de utilizar el marketing digital en la gestión de los procesos universitarios, como vía para mostrar las opciones educativas a los diferentes segmentos de la población, con el apoyo de la web social. El proceso de diagnóstico facilitó el análisis del proceso de marketing y las necesidades de capacitación de los docentes y administrativos.

\section{Bibliografía|}

Arnoldo, H. y Nicolás, M. (2011). Estrategias para el liderazgo competitivo. Buenos Aires:: Editorial Dolmen.

Ayobí, B. (2017). El uso de las redes sociales para la construcción de la imagen corporativa de la empresa decormuebles de montecristi. (Ing. en Comunicación organizacional y Relaciones 
Públicas), Universidad Eloy Alfaro, Manta, Manaí. Ecuador.

Bardo, J. W., Ross, R. H. y Headley, E. L. (2014). "Measuring alumni's image of a university". Journal for Higher Education Management, 6(1), 29-44.

Cordero, L. (2016). Gestión de la imagen corporativa de medias roland utilizando su condición de producto nacional como factor diferenciado. Quito: Universidad de Las Américas.

Dale, R. (2007). "Specifying globalization effects on national policy". The Routledge Falmer Reader in Education Policy and Politics, 14(1), 1-17.

DeShields, O. W., Kara, A. y Kaynak, E. (2005). "Determinants of business student satisfaction and retention in higher education: Applying herzberg's two-factor theory". International journal of educational management, 19(2), 128-139.

Franco, J. y Arrubla, J. (2011). Marketing en universidades. Descripción, análisis y propuestas. Artículo presentado en: Congreso internacional de contaduría administrativa e informática, Colombia.

Hayes, T. (2016). "Delphi study of the future of marketing of higher education". Journal of Business Research, 60(9), 927-931.

Hult, G. T. M. y Ketchen, D. J. (2018). "Does market orientation matter?: A test of the relationship between positional advantage and performance". Strategic management journal of Business Research, 22(9), 899-906.

Kirp, D. L. (2013). Shakespeare, einstein and the bottom line: The marketing of higher education. USA: Harvard University Press.
Kotler, P. y Armstrong, G. (2007). Fundamentos de marketing. México: Pearson Prentice Hall.

Lahuerta, E. y Cordero, R. (2016). "Looking for the perfect tweet. The use of data mining techniques to find influencers on twitter". Computers in Human Behavior(64), 575-583.

Lavrusik, V. (2009). 10 ways universities share information using social media. Fecha de consulta 3-7-2019, 2019, Disponible en: https://www.signe.es/sites/www.signe.es/files leTitulo\%20informe\%20TIC2013.pdf

Muñiz, R. (2010). Marketing xxi. Madrid: Editado por la Universidad a Distancia de Madrid.

Siu, N. Y. y Wilson, R. M. (2017). "Modelling market orientation: An application in the education sector". Journal of Marketing Management, 14(4), 293-323.

Sosa, A. S. y Useche, M. C. (2017). "Marketing digital en universidades privadas en el estado zulia". Editorial Politécnico Grancolombiano, Poliantea, 13(24), 1-29.

Toledo, L. y Luque, T. (2017). "Relación entre marketing y universidad. Revisión teórica y propuesta de un modelo teórico y de marketing 3.0". Revista de Estudios Empresariales. Segunda época(2), 2 - 27.

UNESCO. (1998). Declaración en la conferencia mundial sobre educación superior. Artículo presentado en: Conferencia Mundial sobre Educación Superior, París.

Zapata, E. (2007). Mercadeo educativo. Estrategias para promover instituciones $\mathrm{y}$ programas $\left(2^{\circ}\right.$ ed.). Bogotá: Librería Universitaria. 